\title{
Femtosecond Probe Catches Electrons Relaxing
}

\author{
Pump-probe experiments measure the time it takes for electrons to \\ thermalize and cool after photoexcitation.
}

\section{By Rachel Berkowitz}

A fter being excited by a light pulse, energy carriers in a material scatter off one another to form a hot thermal distribution before relaxing back to equilibrium. Picking apart how these thermalization and relaxation processes occur on ultrashort timescales has been a goal for decades, as they are inherent to phenomena ranging from energy harvesting in solar cells to demagnetization. Hung-Tzu Chang, at the University of California, Berkeley, and his colleagues have now measured the time taken for electrons to thermalize and then cool in a femtosecond-resolution experiment [1]. Such observations are vital to the design of metals and semiconductors for applications in photovoltaics and photocatalysis.

The researchers used 5-fs-long optical laser pulses to excite the electrons in a 50-nm-thick nickel film. After pump-laser illumination, they measured the absorption spectra of the thermalized electrons using sub-4-fs extreme-ultraviolet laser pulses. Energy shifts in the spectral features captured by those pump-probe experiments indicated that it took $13-34 \mathrm{fs}$ for the electrons to thermalize after excitation. The researchers also

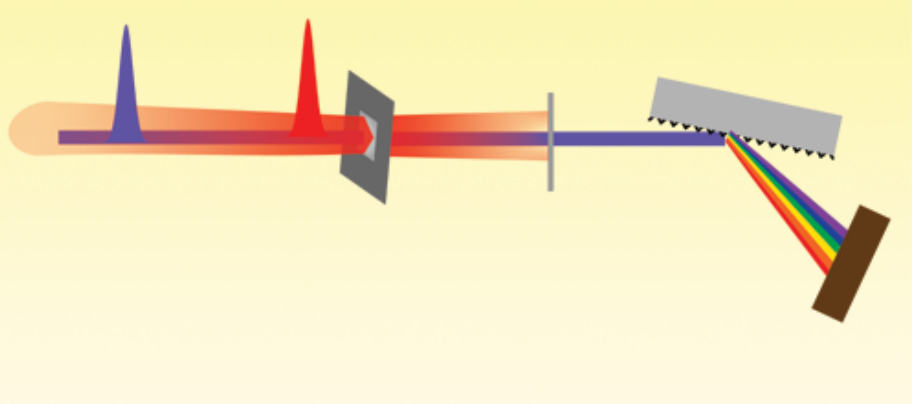

Credit: H.-T. Chang et al. [1] determined that, as these thermalized electrons cooled, their absorption spectra broadened by an amount that was directly proportional to the electron temperature. Tracking this spectral broadening showed that the electrons took $640 \mathrm{fs}$ to cool back to their preexcitation temperature.

Previous studies of photoexcitation have measured electron dynamics on timescales of hundreds of femtoseconds. By observing the thermalization and subsequent cooling of electrons at the sub-20-fs scale, Chang and colleagues have laid the foundation for investigations of ultrafast processes in metals and semiconductors, such as photoinduced phase transitions and electron transport. Such investigations could eventually inform designs of, for example, ultrafast optical switches and magnetic memory.

Rachel Berkowitz is a Corresponding Editor for Physics based in Vancouver, Canada.

\section{REFERENCES}

1. H. Chang et al., "Electron thermalization and relaxation in laser-heated nickel by few-femtosecond core-level transient absorption spectroscopy," Phys. Rev. B 103, 064305 (2021). 